\title{
AN EXTREMAL PROBLEM IN GRAPH THEORY
}

\author{
P. ERDÖS and L. MOSER

\begin{abstract}
To Bernhard Hermann Neumann on his 60th birthday
\end{abstract}
(Received 6 January 1969)

Communicated by G. B. Preston

$G(n ; l)$ will denote a graph of $n$ vertices and $l$ edges. Let $f_{0}(n, k)$ be the smallest integer such that there is a $G\left(n ; f_{0}(n, k)\right)$ in which for every set of $k$ vertices there is a vertex joined to each of these. Thus for example $f_{0}(3,2)=3$ since in a triangle each pair of vertices is joined to a third. It can readily be checked that $f_{0}(4,2)=5$ (the extremal graph consists of a complete 4-gon with one edge removed). In general we will prove: Let $n>\bar{k}$, and

$$
f(n, k)=(k-1) n-\left(\begin{array}{l}
k \\
2
\end{array}\right)+\left[\frac{n-k}{2}\right]+1 ;
$$

then $f_{0}(n, k)=f(n, k)$.

It will be convenient to say that the vertices $x_{1}, \ldots, x_{k}$ of $G$ are visible from $x_{k+1}$, if all the edges $\left(x_{i}, x_{k+1}\right), i=1, \cdots, k$ occur in $G$. A graph is said to have property $P_{k}$ if every set of $k$ of its vertices is visible from another vertex. $G_{n}$ will denote a graph of $n$ vertices (the number of edges being unspecified) and $G(m)$ denotes a graph having $m$ edges. Let $G_{n}^{(0)}=(G n$; $f(n ; k))$ be defined as follows: the vertices of $G_{n}^{(\mathbf{0})}$ are $x_{1}, \cdots, x_{n}$. The vertices $x_{i}, i=1, \cdots, k-1$ are joined to every other vertex and our $G_{n}^{(0)}$ has $[n-k+2 / 2]$ further edges which are as disjoint as possible. In other words if $n-k+1$ is even $G_{n}^{(0)}$ has the further edges $\left(x_{k+2 j}, x_{k+2 j+1}\right), j=0, \cdots$, $[n-k-1 / 2]$, if $n-k+1$ is odd the edges are $\left(x_{k}, x_{k+1}\right),\left(x_{k}, x_{k+2}\right),\left(x_{k+j+1}\right.$, $\left.x_{k+j+2}\right), j=1, \cdots,[n-k-2 / 2]$. It is easy to see that $G_{n}^{(0)}$ has property $P_{k}$. Now we prove

Theorem 1. A graph $G(n ; f(n, k))$ has property $P_{k}$ if and only if it is our graph $G_{n}^{(0)}$.

Theorem 1 is vacuous for $n \leqq k$ and it is trivial for $n=k+1$, thus we can assume $n \geqq k+2$. Clearly Theorem 1 implies (1). To see this it suffices to observe that if a $G(n ; f(n, k)-1)$ would have property $P_{k}$ we could add to it a new edge so that the resulting $G(n ; f(n, k))$ would not be a $G_{n}^{(0)}$. 
Since $G_{n}^{(0)}$ has property $P_{k}$ we only have to prove that a $G(n ; f(n, k))$ has property $P_{k}$ then it must be our $G_{n}^{(0)}$. Before we give the somewhat complicated proof we outline a simple proof of (1) for $k=2$.

Lemma. Let $G_{n}$ have property $P_{k}$ then every pair of its verices is visible from at least $k-1$ vertices.

Assume that the Lemma is false. Then say $x_{1}$ and $x_{2}$ are visible from only $y_{1}, \ldots, y_{l}, l \leqq k-2$. But then the set of $l+2 \leqq k$ vertices $x_{1}, x_{2}, y_{1}, \ldots, y_{l}$ would not be visible from any vertex of $G_{n}$, which contradicts our assumption.

Let now $x_{i}, i=1, \ldots, n$ be the vertices of $G_{n}$ and assume that $v_{i}$ is the valency of $x_{i}$ (i.e. $x_{i}$ is joined to $v_{i}$ vertices of $G$ ). Our Lemma implies

$$
\sum_{i=1}^{n}\left(\begin{array}{l}
v_{i} \\
2
\end{array}\right) \geqq(k-1)\left(\begin{array}{l}
n \\
2
\end{array}\right)
$$

since the number of pairs of vertices visible from $x_{i}$ is $\left(\begin{array}{c}v_{i} \\ 2\end{array}\right)$.

From (2) it is easy to deduce (1) for $k=2$. To see this observe that the number of edges of a graph is $\frac{1}{2} \sum_{i=1}^{n} v_{i}$.

By (2) $\sum_{i=1}^{n}\left(\begin{array}{l}v_{i} \\ 2\end{array}\right) \geqq\left(\begin{array}{l}n \\ 2\end{array}\right)$ and thus by a simple argument $\frac{1}{2} \sum_{i=1}^{n} v_{i}$ will be at least as iarge as in the case that one $v_{i}$ say $v_{1}$ is as large as possible i.e. $v_{1}=n-1$, and $v_{2}, \ldots, v_{n}$ are as small as is consistent with (2). Now it is easy to see that $P_{2}$ implies $v_{i} \geqq 2$ for all $i$. Hence

$$
\frac{1}{2} \sum_{i=1}^{n} v_{i} \geqq \frac{1}{2}(n-1+2(n-1))=\frac{3}{2}(n-1)
$$

which agrees with (1) for $k=2$ if $n$ is odd. If $n$ is even a similar but somewhat more complicated argument proves (1).

It does not seem easy to deduce (1) from (2) for $k>2$. One could easily obtain

$$
f(n, k)=\left(k-\frac{1}{2}\right) n+O(1)
$$

but a more precise estimation seems difficult. Hence to prove (1) and Theorem 1 we shall use a different method.

We say that $G(m)$ has property $\theta_{t}$ if it contains a set $S$ of $t$ vertices $x_{1}, \ldots, x_{t}$ each of which is joined to some vertex of $G(m)$ not in $S . \bar{G}$ is the complementary graph of $G$ i.e. two vertices are joined in $\bar{G}$ if and only if they are not joined in $G$.

Put $n=k+t-1$. Then

$$
\left(\begin{array}{l}
n \\
2
\end{array}\right)-f(n, k)=\left(\begin{array}{l}
t \\
2
\end{array}\right)-\left[\frac{t+1}{2}\right] .
$$

Now a simple argument shows that the fact that $G(n ; f(n, k))$ does not have 
property $P_{k}$ is equivalent to $\bar{G}(n ; f(n, k))=G\left(\left(\begin{array}{l}t \\ 2\end{array}\right)-[(t+1) / 2]\right)$ having property $\theta_{t-1}$. Thus Theorem 1 is equivalent to the following is 'a $\bar{G}_{n}^{(0)}$.

TheOREM 2. Every $G\left(\left(\begin{array}{l}t \\ 2\end{array}\right)-[(t+1) / 2]\right)$ has property $\theta_{t-1}$ except if it

Clearly our $\bar{G}_{n}^{(0)}$ is a $G\left(t,\left(\begin{array}{l}t \\ 2\end{array}\right)-[(t+1) / 2]\right)$ where the missing $[(t+1) / 2]$ edges are as disjoint as possible.

Theorem 2 is vacuous for $t<2$ and trivial for $t \leqq 3$. Henceforth assume $t \geqq 4$.

To prove Theorem 2 let $G\left(\left(\begin{array}{l}t \\ 2\end{array}\right)-[(t-1) / 2]\right)=G$ be any graphs which does not have property $\theta_{t-1}$. We will show that it must be a $\bar{G}_{n}^{(0)}$. First of all we can assume that all vertices of our $G$ have valency $\leqq t-2$. For if not then say $x_{1}$ is joined to $y_{1}, \ldots, y_{t-1}$ which shows that $G$ has property $\theta_{t-1}$ which contradicts our assumption.

Assume next that $G$ has a vertex $x$ of valency $t-2$ (this will be the critical case). Denote by $y_{1}, \ldots, y_{t-2}$ the vertices joined to $x$ and let $z_{1}, \ldots$ be the other vertices of $G$. Clearly no two $z$ 's can be joined. For if $\left(z_{1}, z_{2}\right)$ would be an edge of $G$ then $z_{1}, y_{1}, \ldots, y_{t-2}$ are $t-1$ vertices each of them are joined to a vertex not in the set, or $G$ has property $\theta_{t-1}$. Also no $y$ can be joined to two $z$ 's. For if $y_{1}$ is joined to $z_{1}$ and $z_{2}$ then the $t-1$ vertices $z_{1}, z_{2}, y_{2}, \ldots, y_{t-2}$ would show that $G$ has property $\theta_{t-1}$.

Next we show that at least $t-\mathbf{3} y$ 's are joined to some $z$ (as we know each $y$ can be joined to at most one $z$ ). Assume that $u y$ 's are joined to some $z(u<t-3)$. Clearly $(v(G)$ denotes the number of edges of $G)$

$$
v(G)=\left(\begin{array}{l}
t \\
2
\end{array}\right)-\left[\frac{t+1}{2}\right]=u+\left(\begin{array}{c}
t-1 \\
2
\end{array}\right)-N \text { or } u-N=\left[\frac{t}{2}\right]-1,
$$

where $N$ is the number of the edges of the complete graph spanned by $y_{1}, \ldots, y_{t-2}$ which do not occur in $G$. Now clearly

$$
N \geqq\left[\frac{u+1}{2}\right]
$$

since a $y$ joined to a $z$ cannot be joined to all the other $y$ 's (since otherwise lts valency would be $t-1$ ), hence a missing edge (i.e. an edge not in $G$ ) is incident to every $y$ which is joined to a $z$ and this proves (5). From (4) and (5) we have

$$
\left[\frac{u}{2}\right] \geqq\left[\frac{t}{2}\right]-1
$$

(6) clearly implies $u \geqq t-3$ as stated.

Hence either $u=t-3$ or $u=t-2$. (4) and $u \leqq t-2$ implies that we must have equality in (5) i.e. $N=[(u+1) / 2]$. 
First we prove Theorem 2 if $u=t--3$. (6) implies that if $u=t-3, t$ is odd and since $N=[(u+1) / 2]+[u / 2]=[(t-2) / 2]$ and every $y$ which is joined to a $z$ must be adjacent to a missing edge we obtain that the $[u / 2]$ missing edges must be isolated. In other words we can assume that our $G$ contains all the edges of the complete graph spanned, by $x, y_{1}, \ldots, y_{t-2}$ with the exception of the edges $\left(y_{2 i}, y_{2 i+1}\right), i=1, \ldots,[(t-2) / 2]$. Further every $y_{i}, i=2, \ldots, t-2$ is joined to exactly one $z$. If all these $z$ 's coincide then $G$ is spanned by $x, y_{1}, \ldots, y_{t-2}, z$ and is clearly our $\bar{G}_{n}^{(0)}$ and Theorem 2 is proved in this case.

To complete our proof of the case $u=t-3$ assume that $y_{1}$ is joined to $z_{i}$ and $y_{j}$ to $z_{j},\left(z_{i} \neq z_{j}\right), 2 \leqq i<j \leqq t-2$. But then the $t-1$ vertices $x, z_{i}, z_{j},\left\{y_{l}\right\} 1 \leqq l \leqq t-2, l \neq i, l \neq j$ show that our $G$ has property $\theta_{t-1}$ $\left(x\right.$ and $z_{i}$ are joined to $y_{i}, z_{j}$ is joined to $y_{j}$ and every other $y_{i} l \neq i, l \neq j$ is joined to $y_{i}$ or $y_{j}$ [since the missing edges were isolated]). This contradiction completes the proof of Theorem 2 if $u=t-3$.

Assume next $u=t-2$. Then each $y$ is incident to at least one missing edge and since the number of missing edges is $[(u+1) / 2]=[(t-1) / 2]$ we obtain that for even $t$ there are $(t-2) / 2$ isolated missing edges. Just as in the case $u=t-3$ we see that all the $t-2 y$ 's must be joined to the same $z$. But then we again obtain our $\bar{G}_{n}^{(0)}$. This disposes of the case $u=t-2$, $t$ even.

Assume next $u=t-2, t$ odd. These are $[(t-1) / 2]$ missing edges and since each $y$ is incident to one of them we can assume without loss of generality that the missing edges are $\left(y_{1}, y_{2}\right),\left(y_{1}, y_{3}\right),\left(y_{2 l}, y_{2 l+1}\right), l=2, \ldots$, $[(t-2) / 2]$. If all the $y$ 's are joined to the same $z$ we again get our $\bar{G}_{n}^{(0)}$. Thus we can assume that not all the $y$ 's are joined to the same $z$. Now to complete our proof we have to distinguisn two cases. Assume first that there is a $z$ say $z_{i}$ which is joined to only one $y$ say $y_{i}$. This case can immediately be disposed of since the set of $t-1$ vertices $x, z_{i},\left\{y_{l}\right\}, 1 \leqq l \leqq t-2, l \neq i$ shows that our $G$ has property $\theta_{t-1}\left(x\right.$ and $z_{i}$ are joined to $y_{i}$ and all other $y$ 's are by our assumption joined to a $z$ different from $z_{i}$ ). This contradiction proves Theorem 2 in this case.

Assume finally that every $z$ is joined to more than one $y$ and there are at least two $z$ 's. Let, say, $z_{1}$ be joined to $y_{i}$ and $y_{j}$ and $z_{2}$ to $y_{r}$. Observe now that either every $y$ is joined in $G$ to one of the two vertices $y_{i}$ and $y_{r}$ or every $y$ is joined to one of the two vertices $y_{j}$ and $y_{r}$ (this follows from the fact that the missing edges are either isolated or have at most one vertex of valency two). Assume thus that every $y$ is joined either to $y_{i}$ or to $y_{r}$. But then the set of $t-1$ vertices $x, z_{1}, z_{2},\left\{y_{l}\right\}, 1 \leqq l \leqq t-2, l \neq i, l \neq r$ show that our $G$ has property $\theta_{t-1}\left(x\right.$ and $z_{1}$ are joined to $y_{i}, y_{2}$ to $y_{r}$ and every $y_{l}, l \neq i, l \neq r$ is joined either to $y_{i}$ or $\left.y_{r}\right)$. This contradicticn completes the proof of Theorem 2 if $G$ has a vertex of valency $\geqq t-2$. 
Assume now that all vertices of $G=G\left(\left(\begin{array}{l}t \\ 2\end{array}\right)-[(t+1) / 2]\right)$ have valency $<t-2$. We will show by induction with respect to $i$ that then our $G$ must have property $\theta_{t-1}$ and this will complete the proof of Theorems 2 and 1 and also of (1).

Assume that the maximum valency of a vertex of our $G$ is $r<t-2$. Let $x$ be joined to $y_{1}, \ldots, y_{r}$. Denote as before by $z_{1}, \ldots$ the other vertices of $G$ and let $u$ be the largest number of $z^{\prime}$ s joined to a $y$. Assume that $y_{1}$ is joined to $z_{1}, \ldots, z_{u}$. We evidently have

$$
u \leqq \min (t-r-1, r-1)
$$

To prove (7) observe that $u \geqq r$ would imply $v\left(y_{1}\right)>r$ and $u \geqq t-r$ would imply that $G$ satisfies $\theta_{t-1}$ (consider the vertices $y_{2}, \ldots, y_{r}, z_{1}, \ldots, z_{u}$ ).

Denote by $u_{i}$ the number of $z$ 's joined to $y_{i}\left(u_{1}=u\right)$ and by $w_{i}$ the number of $y$ 's joined to $y_{i}$. By (7) $v\left(y_{i}\right)=1+u_{i}+w_{i} \leqq r-1$. Thus by (7) the number $E$ of edges incident to the vertices $x, y_{1}, \ldots, y_{r}$ equals

(8) $E=r+\sum_{i=1}^{r}\left(u_{i}+\frac{1}{2} w_{i}\right) \leqq r(u+1)+\frac{r(r-u-1)}{2}=\frac{r(r+u+1)}{2} \leqq r^{2}$.

(8) follows from the fact that $E$ is evidently maximal if all the $u_{i}$ are $u=r-1$ (i.e. they are all as large as possible) and if $w_{i}=r-u-1=0$. From (7) we have $\left(G_{1}\right.$ is the graph spanned by the $z$ 's)

$$
v\left(G_{1}\right) \geqq\left(\begin{array}{l}
t \\
2
\end{array}\right)-\left[\frac{t+1}{2}\right]-r^{2} .
$$

Assume first $r \leqq t / 2$. Then we obtain from (9)

$$
v\left(G_{1}\right)>\left(\begin{array}{c}
t-r \\
2
\end{array}\right)-\left[\frac{t-r+1}{2}\right]
$$

Hence by our induction assumption $G_{1}$ has property $\theta_{t-r-1}$ i.e. it contains a set of vertices $z_{1}, \ldots, z_{t-r-1}$ each of which is joined to some $z_{j}$, $j>t-r-1$. But then the $t-1$ vertices $z_{1}, \ldots, z_{t-r-1}, y_{1}, \ldots, y_{r}$ show that $G$ has property $\theta_{t-1}$, which proves Theorem 2 if $r \leqq t / 2$.

Assume next $t / 2<r \leqq t-3$. From (7) we have $u_{i} \leqq t-r-1$ and by (8) $E$ is maximal if all the $u_{i}$ are $t-r-1$ and $w_{i}=r-1-u_{i}=2 r-t$. But then by (8)

$$
E \leqq r+r(t-r-1)+\frac{r}{2}(2 r-t)=\frac{r t}{2} .
$$

From (11) we have

$$
v\left(G_{1}\right) \geqq\left(\begin{array}{l}
t \\
2
\end{array}\right)-\left[\frac{t+1}{2}\right]-\frac{r t}{2}>\left(\begin{array}{c}
t-r \\
2
\end{array}\right)-\left[\begin{array}{c}
t-r+1 \\
2
\end{array}\right]
$$


Thus the proof can be completed as in the previous case, and the proof of Theorem 2 is complete.

Denote by $f_{0}(n, k, r)$ the smallest integer for which there is a. $G\left(n ; f_{0}(n, k, r)\right)$ in which every set of $k$ vertices are visible from at least $r$ vertices. We say that a graph has property $P_{k, r}$ if every set of $k$ of its vertices is visible from at least $r$ vertices. Just as in our Lemma we can show that if $G_{n}$ has property $P_{k, r}$ then every pair of its vertices is visible from at least $k+r-2$ vertices (our old property $P_{k}$ is $P_{k, 1}$ ).

Thus we obtain a.s in (2) that if $G_{n}$ has property $P_{k, r}$ then if $k>1$

$$
\sum_{i=1}^{n}\left(\begin{array}{c}
v_{i} \\
2
\end{array}\right) \geqq(k+r-2)\left(\begin{array}{l}
n \\
2
\end{array}\right) \text {. }
$$

From $\left(2^{\prime}\right)$ we can deduce that if $n<n_{0}(k, r)$ then

$$
f_{0}(n, k, r)=f_{0}(n, k+r-1)=f(n, k+r-1) .
$$

(12) certainly does not hold for every $n, k$ and $r$. It is easy to see that $f_{0}(10,2,6)=40$ but $f(10,7)=41$. Our Theorem 1 states that (12) always holds for $r=1$ and perhaps it always holds for $r=2$ also if $k>1$. For $k=1$ every $G_{n}$ each vertex of which has valency $\geqq r$ clearly has property $P_{1, r}$, thus $f_{0}(n, 1, r)=[(r n+1) / 2]$, in other words if $k=1, r>1$ then (12) is not true. We hope to return to these questions on another occasion.

Finally we can ask the following question: Denote by $F(n, k)$ the smallest integer for which there exists a directed graph $G(n ; F(n, k))$ so that to every $k$ vertices $x_{1}, \ldots, x_{k}$ of our $G$ thete is a vertex $y$ of $G$ so that all the edges $\left(y, x_{i}\right) i=1, \ldots, k$ occur in $G$ and are directed away from $y$. It is easy to see that for $n \geqq 3, F(n, 1)=n$ (for $n \leqq 2$ there clearly is no solution). It is not hard to show that for $n \geqq 7, F(n, 2)=3 n$ and for $n<7$ there is no solution. For $k \geqq \mathbf{3}$, we only have crude inequalities for $F(n, k)$. We say that $G_{n}$ has property $S_{k}$ (after Schütte who posed the problem) if for every set of $k$ nodes $\left(x_{1}, \ldots, x_{k}\right)$ there is at least one node $y$ in $G_{n}$ so that all the edges $\left(y, x_{i}\right), i=1, \ldots, k$ occur in $G$ and are directed away from $y$. Denote by $f(k)$ the smallest value of $n$ for which an $S_{k}$-graph of $n$ vertices exists. We have

$$
(k-1) 2^{k}+3 \leqq f(k)<c k^{2} 2^{k} .
$$

(13) is due to P. Erdös, E. Szekeres and G. Szekeres (Math. Gazette 47 p. 220 and 49 p. 290). We can show that for $n>n_{\mathbf{0}}(k)$

$$
\dot{f(k-1)} \cdot n \leqq F(n, k) \leqq f(k) \cdot n .
$$

University of New South Wales and University of Hawaii 